\title{
Ruling out unresolved binaries in five transitional disks
}

\section{VLT/NACO deep 2.12 and $1.75 \mu \mathrm{m}$ narrow-band imaging}

\author{
S. Vicente ${ }^{1}$, B. Merín ${ }^{2}$, M. Hartung ${ }^{3}$, H. Bouy ${ }^{4}$, N. Huélamo ${ }^{4}$, E. Artigau ${ }^{5}$, J.-C. Augereau ${ }^{6}$, \\ E. van Dishoeck ${ }^{7}$, J. Olofsson ${ }^{8}$, I. Oliveira ${ }^{7}$, and T. Prusti ${ }^{1, \star}$
}

\author{
1 RSSD, European Space Agency (ESTEC), PO Box 299, 2200 AG Noordwijk, The Netherlands \\ e-mail: smvicente@fc.ul.pt \\ 2 Herschel Science Centre, European Space Agency (ESAC), PO Box 78, 28691 Villanueva de la Cañada, Madrid, Spain \\ 3 Gemini Observatory, c/o AURA, Casilla 603, La Serena, Chile \\ ${ }^{4}$ CAB (INTA-CSIC), LAEFF, PO Box 78, 28691 Villanueva de la Cañada, Madrid, Spain \\ 5 Département de Physique and Observatoire du Mont Mégantic, Université de Montréal, C.P. 6128, Succ. Centre-Ville, Montréal, \\ QC H3C 3J7, Canada \\ ${ }^{6}$ UJF-Grenoble 1/CNRS-INSU, Institut de Planétologie et d'Astrophysique de Grenoble (IPAG) UMR 5274, 38041 Grenoble, France \\ 7 Leiden Observatory, Leiden University, PO Box 9513, 2300 RA Leiden, The Netherlands \\ 8 Max Planck Institute for Astronomy, Königstuhl 17, 69117 Heidelberg, Germany
}

Received 10 December 2010 / Accepted 4 June 2011

\section{ABSTRACT}

\begin{abstract}
Context. The presence of unresolved binaries on sub-arsecond scales could explain the existence of optically thin inner holes or gaps in circumstellar disks, which are commonly referred to as "transitional" or "cold" disks, and it is the first scenario to check before making any other assumptions.

Aims. We aim at detecting the presence of companions inside the inner hole/gap region of a sample of five well known transitional disks using spatially-resolved imaging in the near-IR with the VLT/NACO/S13 camera, which probes projected distances from the primary of typically 0.1 to 7 arcsec. The sample includes the stars DoAr 21, HD 135344B (SAO 206462), HR 4796A, T Cha, and TW Hya, spanning ages of less than 1 to $10 \mathrm{Myr}$, spectral types of A0 to K7, and hole/gap outer radii of 4 to 100 AU.

Methods. In order to enhance the contrast and to avoid saturation at the core of the point-spread function (PSF), we use narrow-band filters at 1.75 and $2.12 \mu \mathrm{m}$. The "locally optimized combination of images" (LOCI) algorithm is applied for an optimal speckle noise removal and PSF subtraction, providing an increase of $0.5-1.5$ mag in contrast over the classic method.

Results. With the proviso that we could have missed companions owing to unfavorable projections, the VLT/NACO observations rule out the presence of unresolved companions down to an inner radius of about $0{ }^{\prime} .1$ from the primary in all five transitional disks and with a detection limit of 2 to 5 mag in contrast. In the disk outer regions the detection limits typically reach 8 to 9 mag in contrast and 4.7 mag for T Cha. Hence, the NACO images resolve part of the inner hole/gap region of all disks with the exception of TW Hya, for which the inner hole is only $4 \mathrm{AU}$. The $5 \sigma$ sensitivity profiles, together with a selected evolutionary model, allow to discard stellar companions within the inner hole/gap region of T Cha, and down to the substellar regime for HD 135344B and HR 4796A. DoAr 21 is the only object from the sample of five disks for which the NACO images are sensitive enough for a detection of objects less massive than $\sim 13 M_{\text {Jup }}$ that is, potential giant planets or low-mass brown dwarfs at radii larger than $\sim 76 \mathrm{AU}\left(0{ }^{\prime} 63\right)$.

Conclusions. These new VLT/NACO observations further constrain the origin of the inner opacity cavities to be owing to closer or lower-mass companions or other mechanisms such as giant planet formation, efficient grain growth, and photoevaporation (for DoAr 21 and HR 4796A).
\end{abstract}

Key words. protoplanetary disks - planetary systems - circumstellar matter - stars: general - instrumentation: adaptive optics stars: imaging

\section{Introduction}

The transition from the gas/dust-rich actively accreting optically thick disks surrounding $\mathrm{T}$ Tauri and Herbig Ae/Be stars to the gas/dust-poor optically thin debris disks is currently not well understood. Of the several processes proposed for disk evolution and dissipation, the formation of planets remains the most exciting (e.g., Meyer et al. 2007, and references therein). Early studies of young stars dating back to IRAS (e.g., Strom et al. 1989; Skrutskie et al. 1990) identified several sources with spectral energy distributions (SEDs) that showed

^ Based on observations collected with the NAOS-CONICA (NACO) instrument at the VLT in Paranal Observatory, operated by the European Southern Observatory, under programme ID 079.C-0914(A). a deficit of mid-infrared flux and a rise into the far-IR. These were interpreted as a sign of dust clearing, which is expected from disk evolution as the dust grows and settles or is removed with the gas by viscous accretion or other dispersal mechanisms such as photoevaporation and tidal truncation because of close companions, both stellar and substellar. More recent surveys of young stellar clusters with Spitzer revealed a small population of these sources in several nearby star-forming regions ( $d \leq 400 \mathrm{pc}$ ) based on photometry (Sicilia-Aguilar et al. 2006; Muzerolle et al. 2010), spectroscopy (Brown et al. 2007; Furlan et al. 2009; Oliveira et al. 2010) or both (Sicilia-Aguilar et al. 2008; Merín et al. 2010). They are currently referred to as "transitional" or "cold" disks after Brown et al. (2007), because of their lack of emission from warm dust. The reported 
A\&A 533, A135 (2011)

Table 1. Observing log of the $2.12 \mu \mathrm{m}$ VLT/NACO images of the five stars with transitional disks and corresponding PSF-calibrators.

\begin{tabular}{|c|c|c|c|c|c|c|c|c|c|c|c|c|}
\hline $\begin{array}{l}\text { Star } \\
\& \text { PSF }\end{array}$ & Date & Grade $^{1}$ & $\begin{array}{l}\text { DIT } \\
\text { (s) }\end{array}$ & NDIT & $\begin{array}{l}\text { Exp. time }{ }^{2} \\
\text { (min.) }\end{array}$ & $\begin{array}{l}\text { Seeing }^{3} \\
(\operatorname{arcsec})\end{array}$ & $\begin{array}{l}\text { Airmass } \\
\text { (at start) }\end{array}$ & $\begin{array}{l}\tau_{0}^{4} \\
(\mathrm{~ms})\end{array}$ & $\begin{array}{c}\text { Strehl }^{5} \\
(\%)\end{array}$ & $\begin{array}{r}F W H M^{6} \\
\text { (mas) }\end{array}$ & $\begin{array}{c}V^{7} \\
(\mathrm{mag})\end{array}$ & Notes $^{8}$ \\
\hline $\begin{array}{l}\text { DoAr } 21 \\
\text { CD-24 } 13079\end{array}$ & 2007-06-18 & B & $\begin{array}{c}10.0 \\
4.0 \\
\end{array}$ & $\begin{array}{c}4 \\
1,3\end{array}$ & $\begin{array}{c}8.0 \\
0.73 \\
\end{array}$ & $\begin{array}{l}0.68 \\
0.75\end{array}$ & $\begin{array}{l}1.530 \\
1.547 \\
\end{array}$ & $\begin{array}{l}2.55 \\
2.14\end{array}$ & $\begin{array}{c}9.3 \\
15.2 \\
\end{array}$ & $\begin{array}{c}129.1 \\
85.0\end{array}$ & $\begin{array}{c}14.01 \pm 0.03 \\
9.86\end{array}$ & $\begin{array}{l}\text { sat. } \\
\text { not sat. }\end{array}$ \\
\hline $\begin{array}{l}\text { HD } 135344 \mathrm{~B} \\
\text { HD } 136961\end{array}$ & 2007-05-09 & B & $\begin{array}{l}1.0 \\
1.0 \\
\end{array}$ & $\begin{array}{l}12 \\
10\end{array}$ & $\begin{array}{c}2.4 \\
1.67 \\
\end{array}$ & $\begin{array}{l}1.75 \\
1.85 \\
\end{array}$ & $\begin{array}{l}1.062 \\
1.071 \\
\end{array}$ & $\begin{array}{l}2.73 \\
2.14\end{array}$ & $\begin{array}{l}13.5 \\
14.5\end{array}$ & $\begin{array}{l}82.5 \\
84.6 \\
\end{array}$ & $\begin{array}{c}8.708 \pm 0.017 \\
6.754\end{array}$ & \\
\hline $\begin{array}{l}\text { HD } 135344 \mathrm{~B} \\
\text { HD } 144156\end{array}$ & 2007-06-20 & $\mathrm{B}$ & $\begin{array}{l}2.0 \\
1.0 \\
\end{array}$ & $\begin{array}{c}6 \\
10 \\
\end{array}$ & $\begin{array}{c}2.4 \\
1.67 \\
\end{array}$ & $\begin{array}{l}0.77 \\
0.79\end{array}$ & $\begin{array}{l}1.051 \\
1.076\end{array}$ & $\begin{array}{l}1.07 \\
1.23\end{array}$ & $\begin{array}{l}25.1 \\
26.1 \\
\end{array}$ & $\begin{array}{l}74.4 \\
72.3\end{array}$ & $\begin{array}{c}8.708 \pm 0.017 \\
8.50\end{array}$ & close comp. \\
\hline $\begin{array}{l}\text { HR 4796A } \\
\text { HD } 109536\end{array}$ & 2007-06-14 & $\mathrm{B}$ & $\begin{array}{l}1.0 \\
1.0 \\
\end{array}$ & $\begin{array}{c}10 \\
1 \\
\end{array}$ & $\begin{array}{c}2.0 \\
0.17 \\
\end{array}$ & $\begin{array}{l}\text { NA } \\
\text { NA }\end{array}$ & $\begin{array}{l}1.069 \\
1.092 \\
\end{array}$ & $\begin{array}{l}1.67 \\
1.88 \\
\end{array}$ & $\begin{array}{l}35.9 \\
27.0 \\
\end{array}$ & $\begin{array}{l}69.2 \\
71.9 \\
\end{array}$ & $\begin{array}{c}5.78 \\
5.127 \\
\end{array}$ & \\
\hline $\begin{array}{l}\text { T Cha } \\
\text { CD-78 } 512\end{array}$ & $2007-05-23$ & $\mathrm{~B}$ & $\begin{array}{c}25.0 \\
12.0,2.0\end{array}$ & $\begin{array}{c}2 \\
1,6\end{array}$ & $\begin{array}{c}10.0 \\
1.0,0.8\end{array}$ & $\begin{array}{c}0.85 \\
1.16,1.89 \\
\end{array}$ & $\begin{array}{c}1.735 \\
1.739,1.740\end{array}$ & $\begin{array}{c}1.37 \\
1.28,0.76 \\
\end{array}$ & $\begin{array}{c}4.8 \\
7.8,7.6\end{array}$ & $\begin{array}{c}116.2 \\
97.5,97.5\end{array}$ & $\begin{array}{l}11.86 \\
10.14\end{array}$ & $\begin{array}{l}\text { ellip. } \\
\text { ellip. }\end{array}$ \\
\hline $\begin{array}{l}\text { TW Hya } \\
\text { HD } 94889\end{array}$ & $2007-05-12$ & A & $\begin{array}{l}5.0 \\
1.0 \\
\end{array}$ & $\begin{array}{l}5 \\
5 \\
\end{array}$ & $\begin{array}{c}5.0 \\
0.83 \\
\end{array}$ & $\begin{array}{l}1.46 \\
1.10 \\
\end{array}$ & $\begin{array}{l}1.033 \\
1.053\end{array}$ & $\begin{array}{l}1.27 \\
0.59\end{array}$ & $\begin{array}{l}19.0 \\
23.9\end{array}$ & $\begin{array}{l}78.4 \\
75.7\end{array}$ & $\begin{array}{c}11.07 \\
8.80\end{array}$ & close comp. \\
\hline $\begin{array}{l}\text { TW Hya } \\
\text { HD } 101636\end{array}$ & $2007-05-23$ & B & $\begin{array}{c}5.0 \\
0.3454\end{array}$ & $\begin{array}{l}5 \\
5\end{array}$ & $\begin{array}{c}5.0 \\
0.29\end{array}$ & $\begin{array}{l}0.98 \\
0.86\end{array}$ & $\begin{array}{l}1.020 \\
1.026\end{array}$ & $\begin{array}{l}0.94 \\
0.09\end{array}$ & $\begin{array}{l}18.3 \\
30.8\end{array}$ & $\begin{array}{l}77.9 \\
71.0\end{array}$ & $\begin{array}{c}11.07 \\
9.41\end{array}$ & \\
\hline
\end{tabular}

Notes. ${ }^{(1)}$ The grade of each observing block $(\mathrm{OB})$ is set by the on-site VLT observer based on the user-requested ambient conditions (seeing, sky transparency, airmass) vs. the actual observing conditions. A grade "A" means that the conditions were fully within specifications, "B" that they were mostly within specifications, while " $\mathrm{C}$ " means they were out of specifications and the OB should be repeated if possible; ${ }^{(2)}$ on source $=$ DIT $\times$ NDIT $\times$ number of OBJECT frames; ${ }^{(3)}$ average astronomical site monitor seeing at start in the visible $(0.5 \mu \mathrm{m})$ and at the zenith (airmass $=1$ ). This value can differ significantly from the NACO image quality, which is usually better. "NA" means that no seeing data was available for this night; ${ }^{(4)}$ average coherence time of the atmosphere at $0.5 \mu \mathrm{m}$ calculated by the real time computer (RTC) on real data. Short values of $\tau_{0}(<6 \mathrm{~ms})$ represent a seeing not stable in time; ${ }^{(5)}$ computed with eclipse in the final processed image; ${ }^{(6)}$ of the star in the processed image adopting a Moffat fitting to the radial profile. The full width at half-maximum $(F W H M)$ of the diffraction-limited PSF for the VLT at $2.12 \mu \mathrm{m}$ is 65 mas; (7) optical magnitude retrieved from SIMBAD: 1) DoAr 21 - Cieza et al. (2007), 2) HD 135344B - Høg et al. (2000), 3) HR 4796A Barrado Y Navascués (2006); 4) T Cha - Tanner et al. (2007); 5) TW Hya - Torres et al. (2006); ${ }^{(8)}$ DoAr 21: PSF saturated in the central pixels (4 to 7 pixel range); T Cha: elliptical along $x$-axis; PSF calibrators HD 144156 and HD 94889 have close companions at $d=1^{\prime \prime} 33$, PA $=20^{\circ}$ and $d=2$.' $2, \mathrm{PA}=260^{\circ}$, respectively.

fraction of transitional disks can vary from a few to $50 \%$ depending on definition and the method of classification. A consensus in nomenclature is still lacking and, as demonstrated in Merín et al. (2010), a significant fraction of transitional disks classified with photometry turns out to be not transitional when using spectroscopy. The general definition of a transitional disk is a young disk ( $<10 \mathrm{Myr})$ with an optically thin inner region (the "opacity hole/gap") surrounded by an optically thick outer disk (beyond the hole/gap outer radius, $R_{\text {hole/gap,out }}$ ). The mid-infrared emission originates in the inner edge or "wall" of the truncated disk that is directly illuminated by the star (Calvet et al. 2005). Gapped disks that still retain an inner component of warm optically thick dust, typically of less than a few AU in width, are also referred to as "pre-transitional" after Espaillat et al. (2007). Most transitional disks are still accreting, so that gas is being transported through the inner cleared hole/gap onto the star. In some sources a small amount of micron or sub-micron dust coexists with the gas in the hole/gap region and causes an excess over photospheric fluxes in the near-IR.

The detection of tidal gaps in some disks has confirmed the inference of the inner holes from unresolved data. These gaps and holes have already been resolved with high-resolution contrast imaging and interferometry in the optical, infrared and at (sub)millimeter wavelengths in a handful of objects: LkCa 15 (Thalmann et al. 2010; Piétu et al. 2006), TW Hya (Hughes et al. 2007), LkH 330 (Brown et al. 2008, 2009), GM Aur (Dutrey et al. 2008; Hughes et al. 2009), HR 4796A (Schneider et al. 1999, 2009), SR 21N, WSB 60, DoAr 44, HD 135344B (Andrews et al. 2009; Brown et al. 2009), and T Cha (Olofsson et al. 2011). The direct images and SEDs modeling of transitional disks suggests inner cavities in the dust distribution of
3 to $100 \mathrm{AU}$ in extension (e.g., D'Alessio et al. 2005; Najita et al. 2007; Brown et al. 2007; Cieza et al. 2010; Olofsson et al. 2011). Although several mechanisms have been proposed to explain the origin of inner disk holes or cleared gaps, the existence of previously unresolved close binaries has been confirmed in several transitional disks (Ireland \& Kraus 2008; Pott et al. 2010) and is the first scenario to check before making any other assumptions.

This paper presents near-infrared high-spatial resolution narrow-band imaging of five well known transitional disks DoAr 21, HD 135344B (SAO 206462), HR 4796A, T Cha, and TW Hya - and is aimed at detecting close companions within their inner hole/gap region. The VLT/NACO observations and data reduction are presented in Sect. 2. The results, including the PSF-subtracted images and the sensitivity curves are shown in Sect. 3, where we also refer to the mass limits and orbit constraints of possible companions. In Sect. 4 we tentatively classify the five disks on basis of the mechanisms that potentially create their inner opacity hole/gap. We also analyze the results for each target independently and discuss how the constraints derived from the NACO images complement or support previous studies of companions that made use of other techniques. The conclusions of this study are given in Sect. 5.

\section{Observations and data reduction}

\subsection{Observations}

The target stars were observed with NACO, an adaptive optics supported high-resolution imager and spectrograph installed on ESO's Very Large Telescope in Chile 


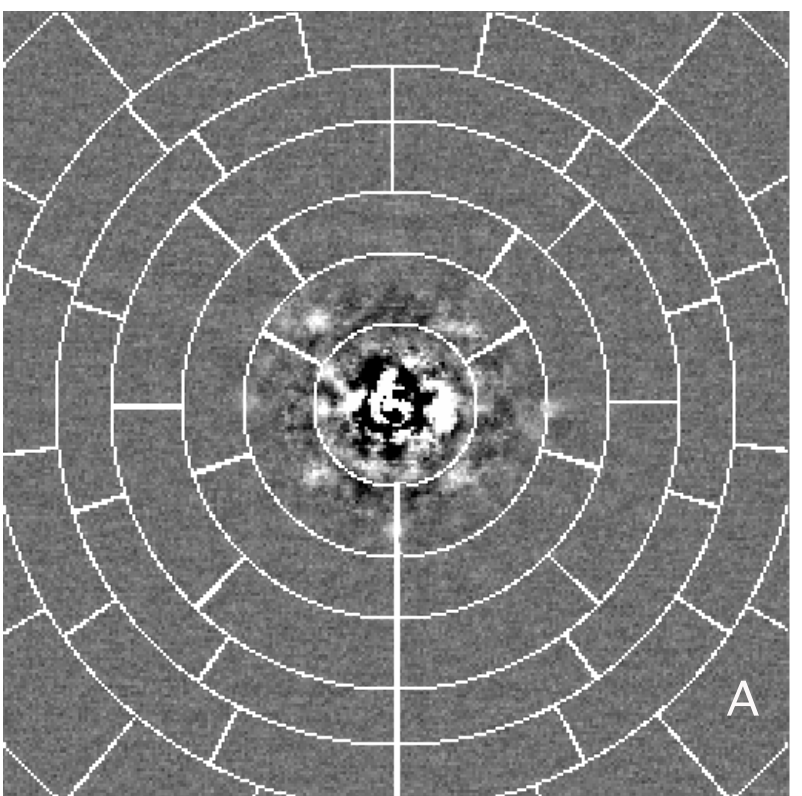

Fig. 1. LOCI optimization grid for a full width at half-maximum $(F W H M)$ of 6 pixels overlaid on the $1.75 \mu \mathrm{m}$ PSF-subtracted image of HD 135344B (09-05-2007). The frame is $512 \times 512$ data pixels (13.27 mas/pixel) and the diameter of the inner circle is $\sim 52$ pixels. The FWHM input used in the LOCI pipeline was adjusted to the average $F W H M$ of the data for each target (for most of the data $F W H M=6$ pixels and for some $F W H M=9$ and 10 pixels). The relevant input parameters for LOCI are not the exact annulus sizes but the surface area of the ring subsections $A$ (see explanation in Sect. 2.2), which in our case corresponds to 70 times the PSF area. The algorithm adjusts the ring sizes to match this area condition (70 PFS cores/segment) and consequently the ring sizes change slightly for different $F W H M$ s and for different radial positions (because the farther out, the more segments).

(Rousset et al. 2003; Lenzen et al. 2003). The observations were performed in service mode in several runs between May and June 2007 with two narrow-band filters, NB_2.12 $(2.122 \pm 0.011 \mu \mathrm{m})$ and NB_1.75 $(1.748 \pm 0.013 \mu \mathrm{m})$, to avoid saturation in the core of the PSF. The images were collected with the highest spatial resolution camera S13 (13.27 mas/pixel, $14^{\prime \prime} \times 14^{\prime \prime}$ FoV) and with the NAOS visible wavefront-sensor and the visible dichroic. The camera "Double RdRsRd" readout mode and the "HighDynamic" mode (15000 ADU Full Well Depth) were selected to observe these bright stars. The PSF standard stars were chosen to be close in spectral type and parallactic angle to the target stars. They were observed immediately after the science targets to match sky conditions as well as possible. More details on the observations can be found in Table 1. The observing conditions of the different runs were mostly poor (bad and highly variable seeing, clouds, winds), resulting in a low adaptive optics (AO) performance. The analyzed datasets barely met the required specifications; part of the data had to be discarded. Only one dataset was fully within specifications. Hence, future datasets obtained under more favorable atmospheric conditions will certainly further improve the results.

\subsection{Data reduction and the $\mathrm{LOCl}$ algorithm}

We adopt a strategy different from the "classic" PSF subtraction approach that takes advantage of the stability of the quasi-static speckle pattern of the AO-corrected PSF. Each individual image (DIT) of the target is dark-subtracted and flat-fielded using standard reduction procedures with IRAF and the NACO pipeline, which is based on the eclipse library (Devillard 1997). For each of them, the stellar PSF speckles are attenuated by subtracting an optimized PSF obtained using the "locally optimized combination of images" (LOCI) algorithm detailed in Lafrenière et al. (2007). Conceptually, this data processing technique uses the fact that high-contrast imaging PSFs from a given instrument - even when taken with different observational setups - share common speckle structures. If an observing strategy is arranged in a way that a set of images with similar speckle patterns can be taken while a faint companion is present in one image and absent in others, one can build a "best estimate" of the PSF for a given image from the set of reference images (PSF images). This "best estimate" is subtracted from the target frame and a significant gain in sensitivity can be achieved.

This approach was initially conceived in the context of angular differential imaging or ADI (see demonstration in Fig. 8 of Lafrenière et al. 2007; and its comparison with the Marois et al. 2006, ADI results), where a long sequence of observations is taken with the Cassegrain rotator turned "off". Through the sequence, a companion at a given field position will rotate through the field as the parallactic angle changes but the average speckle pattern caused by telescope and instrument aberrations typically remains stable. With this sequence, a high-precision PSF frame can be built for each image frame from all other frames taken at sufficiently different angular positions (to avoid self-subtraction of a companion). This technique can be extended to any set of images where a putative companion is absent or significantly suppressed $^{1}$. That means it can also be applied to a conventional data set (field rotation compensated for by the Cassegrain rotator following) using PSF stars that are separately recorded as we do in this paper.

The heart of the LOCI algorithm can be described quite easily: a specific target frame is divided into "optimization subsections" and the best linear combination of reference images is found for every optimization subsection to represent the corresponding target frame section. The idea to split the target image into subsections takes into account that the correlation between target and reference (PSF) images typically varies with its field position. A crucial step in the LOCI algorithm is the definition of a proper set of reference images. In angular differential imaging data for e.g., the displacement caused by field rotation has to be large enough to avoid self-cancellation. This complication does not exist in our case. We simply used another set of PSF star observations with an identical instrumental setup. But we exploit the fact that NACO PSFs show a strong degree of mirror symmetry and also add the mirror-flipped versions of each PSF frame to the set of reference frames ${ }^{2}$. For the NACO data we adjusted the surface area of the optimization subsections $A$ (as seen in the grid in Fig. 1) to contain approximately 70 "PSF cores", i.e., we set Lafrenière's parameter $N_{A}$ to 70 in Eq. (1) of Lafrenière et al. (2007):

$$
A=N_{A} \pi\left(\frac{F W H M}{2}\right)^{2}
$$

${ }^{1}$ E.g. by spectral differential imaging (ADI). See in Artigau et al. (2008) the application of LOCI to the Gemini Near-Infrared Coronographic Imager (NICI) data, a dual-channel imager exploiting the methane absorption feature for planet search.

${ }^{2}$ Note that obviously LOCI provides a higher gain in sensitivity for ADI data because there is no additional slew to acquire the PSF star and furthermore the pupil does not rotate. Therefore, there is a better match of the quasi-static speckle pattern for the reference and target frames. 




Fig. 2. High-contrast residual images after PSF removal with the LOCI algorithm for both filters NB_1.75 and NB_2.12. Each panel is a 3"'3 $\times$ 3". 3 sub-image centered on the target star. The bottom set of panels is a zoom-in of the inner $1^{\prime \prime} \times 1$ " region for the NB_2.12 filter showing the PSF residuals at small distances from the primary. No speckles were found to be achromatic (i.e., no evidence for a real object) and present in the two epochs of HD 135344B ( 1.5 month apart) and TW Hya (just a few days apart) for which only the best dataset is displayed. North is up and east to the left.

where the $F W H M$ is the full width at half-maximum of the PSF and $N_{A}$ the number of "PSF cores" that fit in the optimization subsection. We did not pursue an extensive optimization for this parameter but kept it large enough for convenient calculation times and to be on the safe side concerning self-cancelation. Figure 1 shows the annulus sizes and geometry of the optimization subsections $A$ for a $F W H M$ of 6 pixels.

After subtracting the reconstructed PSF from every frame, the images were registered and median-combined. The remaining speckles add up incoherently in the final image, while any companion would add up coherently. The final images or PSF-subtraction residuals are shown in Fig. 2.

\section{Results}

Figure 2 shows the final images after the data reduction and analysis with the LOCI high-contrast technique described above. Each tile $\left(3^{\prime \prime} .3 \times 33^{\prime \prime} 3\right)$ is centered on the target star and each row displays the residual images in one of the two narrowband filters. The bottom set of panels is a zoom-in of the inner $1^{\prime \prime} \times 1^{\prime \prime}$ region for the NB_2.12 filter. The optimal suppression of speckle noise and the improvement in the sensitivity limit allows us to search for eventual companions. Some speckles remain in the images after LOCI and are caused by the nonoptimal observational strategy. Electronic ghosts aligned with the $X$ and $Y$ axis and row saturation effects typical of NACO were present in many of the processed frames of the target stars and PSF-calibrators. These residuals can be discarded by blinking between the 1.75 and $2.12 \mu \mathrm{m}$ images, or between different datasets if they were acquired close in time and hence are not affected by the orbital motion of a possible companion. A real companion looks like a speckle that does not move between the different filters (achromatic) or different datasets when available, as in the case of TW Hya (just a few days difference) and HD 135344B ( 1.5 month). A careful visual analysis of the images discarded any achromatic speckles that could point to a tentative detection of nearby sources. The best images of HD 135344B (20 June 2007) and TW Hya (12 May 2007) are shown. We note that even though our data are not ideal for LOCI application (poor AO performance, low contrast, and speckles are less dominant), the results are still significantly better than those obtained with "classic" PSF subtraction (see Fig. 3).

\subsection{Detection and mass limits for putative stellar/substellar companions}

In order to quantify the upper limits on mass of potential companions, we compute the differential magnitude profile as a function of the separation from the primary star. They are obtained by calculating the $5 \sigma$ rms over concentric annuli ( $1 F W H M$ width) of the intensity on the residual maps (PSF-subtracted) displayed in Fig. 2. These resulting sensitivity curves are shown for each filter and image in Fig. 3. The black and pink lines represent the differential magnitude curves for the NB_1.75 and NB_2.12 filters, respectively. For comparison, the dashed curve shows the $5 \sigma$-sensitivity not applying LOCI but standard PSF subtraction to the $2.12 \mu \mathrm{m}$ images. The LOCI algorithm improves the detection sensitivity by 0.5 up to 1.5 mag which corresponds to an improvement of up to factor of 4 in contrast ratio. The projected separation in AU was computed using the distance to the stars from Table 2 and is displayed at the bottom $x$-axis. The separation in arcsec is shown at the top $x$-axis. We typically reach a contrast of 2 to $5 \mathrm{mag}$ at $0^{\prime} .1$ and 4 to $6 \mathrm{mag}$ at 0.2 . The mass limits can be estimated with a selected evolutionary model of a substellar object. The cyan and green lines mark the approximate positions of the canonical values of 75 and $13.7 M_{\text {Jup }}$ 
S. Vicente et al.: Ruling out unresolved binaries in five transitional disks
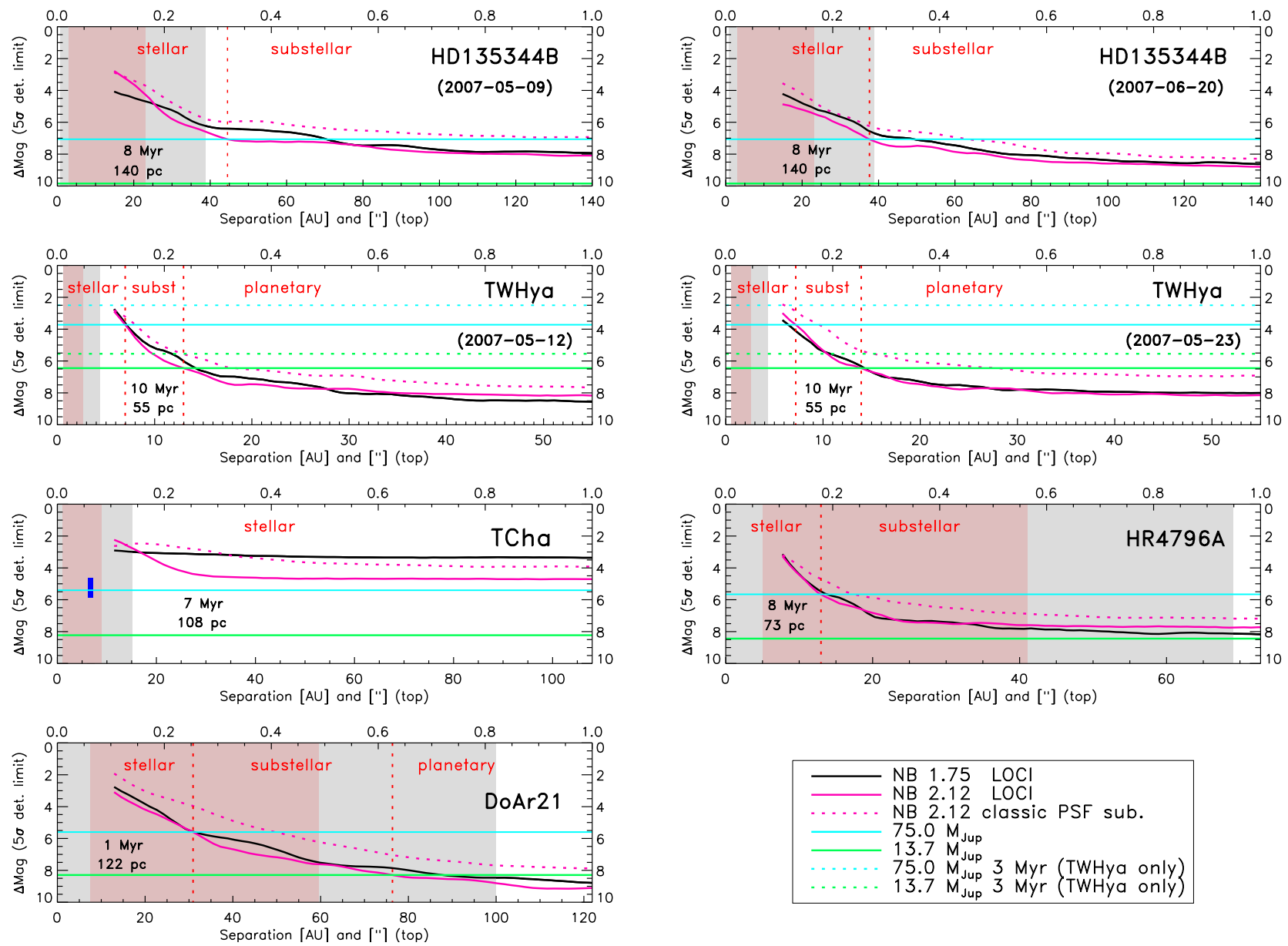

Fig. 3. $5 \sigma$-sensitivity profiles as a function of the distance from the star. The NB_1.75 and NB_2.12 filters are shown in black and pink lines, respectively. For comparison, the dashed curve shows the $5 \sigma$-sensitivity for standard PSF-subtracted images at $2.12 \mu \mathrm{m}$, not using LOCI. The cyan and green lines mark the approximate positions of 75 and $13.7 M_{\text {Jup }}$, the canonical values that define the substellar and planetary regime boundaries according to the BT-DUSTY models from Allard et al. (2010). The dashed lines represent the same boundaries but for an age of 3 Myr for TW Hya recently reported by Vacca \& Sandell (2011). The gray areas show the disk inner hole/gap sizes reported in the literature and the red dashed vertical lines mark the intersections of the LOCI contrast curve at $2.12 \mu \mathrm{m}$ with the cyan and green lines. They indicate the radial distance from the primary from where the NACO images are sensitive to substellar and planetary mass objects according to the adopted BT-DUSTY model. The pink areas represent the hole/gap distance range where stellar companions could exist according to the models of Artymowicz \& Lubow (1994) on basis of the hole/gap size. For typical gaseous viscous disk parameters, and $a$ denoting the binary semi-major axis, the inner edge location of the circumbinary disk varies from $1.7 a$ to $3.3 a$ for binary eccentricity increasing from 0 (circular orbit) to 0.75 , and assuming a face-on geometry $\left(i=0^{\circ}\right.$, PA $=0^{\circ}$ ). The position of the companion varies from $a(1-e)$ and $a(1+e)$, the periapsis and apoapsis of the secondary relative to the primary. For TCha, the NACO images are only sensitive to stellar mass objects brighter than $4.7 \mathrm{mag}$ in contrast or $0.12 M_{\odot}$. The blue vertical bar in the TCha plot indicate the approximate $5-\sigma$ detection limit $\left(\Delta K_{\mathrm{s}}>4.65 \mathrm{mag}\right.$ or mass $\left.<0.12 M_{\odot}\right)$ at the separation $(6.7 \pm 1 \mathrm{AU})$ and position angle $(\sim 78 \pm 1 \mathrm{deg})$ of the companion candidate detected in the $L^{\prime}$-band with NACO by Huélamo et al. (2011). A lower limit of $50 M_{\text {Jup }}$ was chosen following Fig. $4 \mathrm{~b}$ in their paper. This detection is well within the disk hole/gap of T Cha and inside the binary range predicted by Artymowicz \& Lubow (1994). The profiles are displayed between 0.1 and 1 arcsec for all stars. Simulations with a fake target of 4 mag in contrast show that the sensitivity curves are reliable as close as 100-120 mas from the primary (between the first and second Airy ring at $2.12 \mu \mathrm{m}$ ). Beyond 1 arcsec and up to 3.3 arcsec the contrast curves remain flat.

(Burrows et al. 1997, 2001) that define the substellar and planetary regime boundaries ${ }^{3}$ for comparison with our data. They

\footnotetext{
3 The mass boundaries between stars, brown dwarfs, and planets are model-dependent. The former is defined as the minimum mass required for burning hydrogen for a solar metallicity object in the mainsequence. This value is a function of the helium fraction, metallicity and opacity of grains and, depending on the assumed parameters, it can range from 0.07 to $0.092 M_{\odot}$ or 73 to $96 M_{\text {Jup }}$ (Burrows et al. 1997, 2001, and references therein). The latter is defined as the mass-limit for deuterium burning and varies with the helium abundance, the initial deuterium abundance, the metallicity and the fraction of the initial deuterium abundance that must combust for the substellar object to qualify as having burned deuterium (Spiegel et al. 2011). The deuterium
}

were computed by interpolating the mass vs. NB_ 2.12 relation of the BT-DUSTY models from Allard et al. $(2010)^{4}$ convolved with the NACO filter at the distances and ages listed in Table 2.

mass limit can range from $\sim 11.0$ to $\sim 16.3 M_{\text {Jup }}$ depending on the model assumptions. A final remark is that other criteria, such as the formation process, should be considered when classifying a substellar object.

4 These are the models of brown dwarfs and very low-mass stars with dusty atmospheres from Allard et al. (2001) and Chabrier et al. (2000) but with updated opacities. One should be cautious when applying evolutionary models developed for stars and substellar objects to derive the mass of planets. The mass of a freshly formed planet only depends on the accretion history and accretion rate and a planetary model should be considered instead. 
Table 2. Physical properties of the five stars and their disks found in the literature.

\begin{tabular}{|c|c|c|c|c|c|c|c|c|}
\hline Star & SpT & $K_{\mathrm{s}}{ }^{1}$ & $\Delta K_{\mathrm{s}}{ }^{1}$ & $\begin{array}{l}\text { Dist. } \\
\text { (pc) }\end{array}$ & $A_{V}$ & $\begin{array}{l}M_{\text {star }} \\
\left(M_{\odot}\right)\end{array}$ & $\begin{array}{l}\text { Age } \\
(\mathrm{Myr})\end{array}$ & $\begin{array}{c}\dot{M}_{\mathrm{acc}^{2}} \\
\left(M_{\odot} \mathrm{yr}^{-1}\right)\end{array}$ \\
\hline DoAr 21 & $\mathrm{~K} 1^{a}$ & 6.227 & 0.018 & $121.9 \pm 5.8^{b}$ & $6.2^{a}$ & $1.8^{a}$ & $<1^{a}$ & $<10^{11, c}$ \\
\hline HD 135344B & $\mathrm{F} 4^{e}$ & 5.843 & 0.020 & $140 \pm 42^{g}$ & $0.5^{e}$ & $1.7 \pm 0.2^{g}$ & $8 \pm 4^{g}$ & $5 \times 10^{-9 g}$ \\
\hline HR 4796A & $\mathrm{A} 0^{i}$ & 5.769 & 0.023 & $72.8 \pm 1.7^{h}$ & - & $2.5^{i}$ & $8 \pm 2^{i}$ & - \\
\hline TCha $^{6}$ & $\mathrm{G} 8^{j, k}$ & 6.954 & 0.018 & $108 \pm 9^{l}$ & $1.7^{k, 5}$ & $1.5^{j}$ & $7^{k, 5}$ & $4 \times 10^{-9 k}$ \\
\hline $\mathrm{TW} \mathrm{Hya}^{7}$ & $\mathrm{~K} 7^{\circ}$ & 7.297 & 0.024 & $55 \pm 9^{h}$ & $0.0^{q}$ & $0.6^{r}$ & $10^{p}, 3^{*}$ & $8 \times 10^{-10 q}-10^{-9 y}$ \\
\hline \multirow[t]{2}{*}{ Disk } & $R_{\text {disk,in }}{ }^{3}$ & $R_{\text {hole/gap,in }}{ }^{3}$ & $R_{\text {hole/gap,out }}{ }^{3}$ & $R_{\text {disk,out }}{ }^{3}$ & $i$ & PA & $M_{\mathrm{disk}}{ }^{4}$ & Class \& disk type ${ }^{5}$ \\
\hline & $\begin{array}{l}\text { SED, obs. } \\
\text { (AU) }\end{array}$ & $\begin{array}{l}\text { SED, obs. } \\
\text { (AU) }\end{array}$ & $\begin{array}{l}\text { SED, obs. } \\
\text { (AU) }\end{array}$ & $\begin{array}{l}\text { SED, obs. } \\
\text { (AU) }\end{array}$ & (deg) & (deg) & $\left(M_{\odot}\right)$ & \\
\hline DoAr 21 & - & - &,$- 100^{a}$ & - & $<45^{d}$ & - & $<0.001^{c}$ & PMS circumbinary/debris ${ }^{c}$ \\
\hline HD 135344B & $0.18^{e}, 0.05 \pm 0.25^{w}$ & $0.45^{e}, 1.8 \pm 0.2^{w}$ & $45^{e}, 39 \pm 4^{f}$ & $300^{e}, 160^{g}$ & $21^{f}$ & $55^{f}$ & $(2.8 \pm 1.3) \times 10^{-3, g}$ & PMS Herbig ${ }^{g}$ \\
\hline HR 4796A & $\begin{array}{c}0.10,0.00 \\
-\end{array}$ & $\begin{array}{c}0.72,1.0+0.2 \\
-\end{array}$ & $40-200^{i}, 69^{i}$ &,$- 87^{i}$ & $75.88 \pm 0.16^{i}$ & $27.01 \pm 0.16^{i}$ & $\geq 7.4 \times 10^{-5, i}$ & Herbig debris disk ${ }^{i}$ \\
\hline $\mathrm{TCha}^{6}$ & $0.08^{e} 0.13^{n},-$ & $0.2^{e} 0.17^{n},-$ & $15^{e} 7.5^{n},-$ & $300^{e, n},-$ & $75^{j}, 60^{n}$ & $78_{-50}^{+87 n}$ & $0.003^{m},(1.76 \pm 0.25) \times 10^{-2, n}$ & $\mathrm{WTT}^{z}$ \\
\hline $\mathrm{TW} \mathrm{Hya}^{7}$ & $\sim 0.02^{r},-$ & $0.25^{t}, 0.06^{u}$ & $4^{r}, 4.3 \pm 0.3^{s}$ & $230^{x}$ & $4.3 \pm 1.0^{v, 1}, 7 \pm 1^{v, 2}$ & $332 \pm 10^{v}$ & $\sim 0.06^{r}$ & CTT $^{*}$ \\
\hline
\end{tabular}

Notes. ${ }^{(1)}$ From 2MASS; ${ }^{(2)}$ average accretion rate derived from $\mathrm{H} \alpha$ line emission for DoAr 21 and T Cha, Br $\gamma$ line emission for TW Hya (y) and HD 135344B (g), and excess Balmer continuum for TW Hya (q); ${ }^{(3)}$ radius from the primary derived from both SED fitting models and spatially resolved observations in the optical to the sub-mm/mm. In this work we adopt the latter values when available; ${ }^{(4)}$ total disk masses estimated from mm fluxes assuming a gas-to-dust ratio of 100 or 77 for TW Hya. TCha: the first value is that of the 3 mm emission flux for a distance of 66 pc. Because $M_{\text {disk }} \propto d^{2}$, a value of $0.008 M_{\odot}$ is expected for the assumed distance $d=108$ pc. The second value was derived from model fitting to the observed SED and $H$ and $K$-band interferometric observations with the VLTI/AMBER instrument; ${ }^{(5)}$ young stellar object (YSO) evolutionary class and disk type found in the literature: pre-main sequence (PMS), weak lined T Tauri (WTT) and classical T Tauri (CTT) star; (6) T Cha shows variable circumstellar extinction $A_{v}=1.2-4.6$ and an age of 4.1-10 Myr (Schisano et al. 2009, and references therein). Here we adopt the most frequent value $A_{v}=1.7$ and age of 7 Myr. The disk inner hole size adopted is from Brown et al. (2007); (7) a recent work by *Vacca \& Sandell (2011) based on near-infrared spectroscopy revises the spectral type and age of TW Hya to M2.5 (instead of K7) and 3 Myr (instead of 8-10 Myr). Akeson et al. (2011) combines new near-IR interferometric data with previous spatially resolved observations at $10 \mu \mathrm{m}$ and $7 \mathrm{~mm}$ to constrain disk models based on a flared disk structure. They find TW Hya fits a three-component model composed by an optically thin emission from $\sim 0.02 \mathrm{AU}$ to $\sim 0.5 \mathrm{AU}$, an optically thick $\sim 0.1 \mathrm{AU}$ wide ring that is followed by an opacity gap, and an outer optically thick disk starting at 3.8-4.5 AU. Here we adopt the values of 0.5 AU and 4.3 AU for the inner and outer hole/gap radius, respectively.

References. ${ }^{(a)}$ Jensen et al. (2009); ${ }^{(b)}$ Loinard et al. (2008); ${ }^{(c)}$ Cieza et al. (2010, 2008); ${ }^{(d)}$ Bary et al. (2002); ${ }^{(e)}$ Brown et al. $(2007) ;(f)$ Brown et al. (2009); ${ }^{\left({ }^{)}\right)}$Grady et al. (2009) and references therein; ${ }^{(h)}$ van Leeuwen (2007); ${ }^{(i)}$ Schneider et al. (1999, 2009), Jura et al. (1995), Besla \& $\mathrm{Wu}$ (2007) and references therein; ${ }^{(j)}$ Alcala et al. (1993); ${ }^{(k)}$ Schisano et al. (2009); ${ }^{(l)}$ Torres et al. (2008); ${ }^{(m)}$ Lommen et al. (2007); ${ }^{(n)}$ Olofsson et al. (2011); ${ }^{(o)}$ Herbig (1978); ${ }^{(p)}$ Webb et al. (1999); ${ }^{(q)}$ Herczeg et al. (2009); ${ }^{(r)}$ Calvet et al. (2002); ${ }^{(s)}$ Hughes et al. $(2007) ;{ }^{(t)}$ Thi et al. (2010); ${ }^{(u)}$ Eisner et al. (2006); ${ }^{(v, 1)}$ Pontoppidan et al. (2008) for the inner disk; ${ }^{(v, 2)}$ Qi et al. (2004) for the outer disk; ${ }^{(w)}$ Fedele et al. (2008); ${ }^{(x)}$ Weinberger et al. (2002); ${ }^{(y)}$ Eisner et al. (2010); ${ }^{(z)}$ Alcala et al. (1993).

We then subtracted the 2MASS $K_{\mathrm{s}}$ absolute magnitudes of the stars (at the assumed distance and corrected for extinction using the interstellar extinction law of Rieke \& Lebofsky 1985 with $R_{V}=3.1$ ) to obtain the difference in magnitude expected for a companion of 13.7 and $75 M_{\text {Jup }}$. The NB_ 2.12 filter was selected because a better contrast is expected at longer wavelengths. The approximation of NB_2.12 mag $\sim K_{\mathrm{s}}$ mag is a good first estimate (within $\leq 0.1 \mathrm{mag}$ ) that does not compromise the results if we account for all the errors involved. A younger age for TW Hya of 3 Myr (instead of 8-10 Myr) has been reported in Vacca \& Sandell (2011). We computed the substellar and planetary mass boundaries of TW Hya for an age of $3 \mathrm{Myr}$ and plotted it as cyan and green dashed lines for comparison. For this case, the NACO images are sensitive to substellar mass objects within

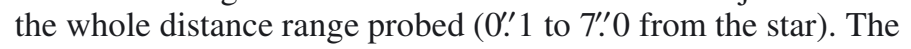
gray area in the plots represents the size of the inner hole/gap determined from previous studies (Table 2). Three of the five disks in the sample have an optically thick inner ring of sub- to nearly $2 \mathrm{AU}$ in radii reported in the literature. Here we adopt the inner hole/gap radii of 1.8 AU for HD 135344B (Fedele et al. 2008), 0.2 AU for T Cha (Brown et al. 2007) and 0.6 AU for TW Hya from the recent results of Akeson et al. (2011). Nevertheless, these values are not relevant for the analysis in this paper because the NACO observations are not sensitive to these inner radii. The vertical red dashed lines give an indication of the disk distance range where the images are sensitive to the substellar and planetary regimes, according to the BT-DUSTY model adopted here. The inner 100 mas of the profiles is omitted. The most inner central region of the residual maps is not dominated anymore by speckle or other noise but by systematic errors in removing the PSF core. In this region the "standard" contrast curve calculation does not apply anymore. Simulations with a 4 mag contrast fake target show that the contrast curves are reliable to about the inner 100-120 mas or between the first and second Airy ring at $2.12 \mu \mathrm{m}$. Table 2 lists the physical parameters of the five stars and their disks found in the literature.

\subsection{Orbit constraints on putative stellar/planetary companions}

This paper addresses one possible origin for inner holes or cleared gaps in protoplanetary disks, which is the dynamical clearing by unresolved companions. In this section, we discuss the expected orbits of potential companions that account for the observed hole/gap size on basis of existing models or numerical simulations for both planets (Quillen et al. 2004; Varnière et al. 2006) and stars (Artymowicz \& Lubow 1994).

We caution that these quantitative estimates are very uncertain because we do not know the orbital parameters and only poorly constrain the disk inclinations and other properties. The 
important result in this paper is qualitative and refers to the presence or absence of stellar/substellar companions beyond the 0 .' 1 radius probed with the NACO images. We cannot completely rule out companions as long as there is a small chance of alignment during the observations that could have occulted the companion. But the likelihood of that happening is small. Table 3 shows the results for the distance range around each of the stars where we can effectively rule out the presence of stellar and substellar companions on the basis of our NACO observations and with the limitation that we could have missed companions because of unfavorable projections.

\subsubsection{Planetary companions}

A single planet of a few $M_{\text {Jup }}$ carves only a narrow gap (typically $\Delta r_{\text {gap }} / r_{\text {gap }} \sim 0.1$ ) about its orbit in a viscous disk that is a few times more massive than the planet and as soon as the Hill's radius ${ }^{5}$ is larger than the disk height (e.g., Lubow $\&$ D'Angelo 2006). This is not compatible with the large inner hole/gap observed in most transitional disks. Nevertheless, a system of multiple planets could open large holes and maintain sharp inner disk edges (Perez-Becker \& Chiang 2011, and references therein). Another possibility is to consider that the mass of the single planet is large compared to the disk mass with which it interacts so that the inertia of the planet slows the migration and the inner disk within the planet's semi-major axis has time to viscously accrete onto the star, creating a large inner hole (Quillen et al. 2004). The material beyond the planet's orbital radius is prevented from accreting by the transfer of orbital angular momentum from the planet to the disk and piles up, forming the inner rim of the outer disk. Additionally, if the massive planet forms faster than the migration timescale and slower than the hole/gap at its formation radii, numerical simulations by Varnière et al. (2006) show that the "spectral hole" always accompanies the planet and extends from the planet's orbital radius to the stellar surface. Here "spectral hole" subtends a region with reduced density, which is not necessarily fully depleted of material, allowing for accretion to hold even after the hole has formed. If the planet forms faster than the hole formation timescale, a gap can open with an inner radius larger than the star radius and an outer edge beyond the planet's orbital radius. Other mechanisms such as outward migration (Masset \& Papaloizou 2003) could contribute to a gap opening beyond the planet's orbital radius. In this paper, and for simplicity, we assume that a planet of a few $M_{\text {Jup }}$ could exist within the whole extension of the hole/gap region of the five transitional disks in our sample.

\subsubsection{Stellar companions}

Binary systems with separations smaller than the outer disk, on the other hand, are expected to open inner holes that are significantly larger than the semi-major axis of the orbit of the binary, while keeping circumprimary disks that dissipate quickly on viscous timescales. In this case, the inner hole or gap is created by tidal truncation of the disk which, initially interpreted as transitional, is in fact a circumbinary disk. Artymowicz \& Lubow (1994) investigated the gravitational interaction of an eccentric binary with the circumbinary and circumstellar gaseous disks and analytically computed the approximate size of the

\footnotetext{
5 The Hill radius of a planet is given by $R_{\mathrm{H}}=a_{\mathrm{p}}(1-e)\left(\frac{m_{\mathrm{p}}}{3 M_{\star}}\right)^{1 / 3}$, where $a_{\mathrm{p}}$ is the semi-major axis of the orbit of the planet, $e$ the eccentricity, $m_{\mathrm{p}}$ the mass of the planet and $M_{\star}$ the mass of the star.
}

disk gaps as a function of the binary mass ratio and eccentricity. For typical viscous disk parameters and $a$ denoting the semi-major axis of the binary, the inner edge location of the circumbinary disk relative to the center of mass of the binary, or $R_{\text {hole,out }}$, varies from $1.7 a$ to $3.3 a$ for a binary eccentricity increasing from 0 (circular orbit) to 0.75 and assuming a face-on geometry $\left(i=0^{\circ}\right.$, PA $\left.=0^{\circ}\right)$. Therefore, $R_{\text {hole, out }} / 3.3<a<$ $R_{\text {hole,out }} / 1.7$ and the position of the companion $d_{\mathrm{S}}$ varies from $a(1-e)$ to $a(1+e)$, the periapsis and apoapsis of the secondary relative to the primary. Observations of binary systems inside disk holes suggest that these numbers are approximately correct (e.g., Guenther et al. 2007, for CS Cha; Ireland \& Kraus 2008, for Coku Tau/4; Beust \& Dutrey 2006, for GG Tau; and more recently, Huélamo et al. 2011, for T Cha). The circumbinary disk recedes from the binary with increasing eccentricity. For simplicity, we assume the origin of the coordinate system to be coincident with the primary star. This will not affect the results for $d_{\mathrm{S}}$ significantly because the clearing is always larger than $a$ and because of the many uncertainties involved.

In order to compute the expected positions of stellar companions for the stars in our sample, we considered the example in Artymowicz \& Lubow (1994) of a binary star with viscosity parameter $\alpha_{v} \sim 10^{-2}$, Reynolds number $R \sim 10^{5}$ and binary mass parameter $\mu=0.3$ for which $R_{\text {hole,out }}=1.7 a$ for $e=0$ (model 1$), R_{\text {hole, out }}=2.6 a$ for $e=0.25$ (model 2$)$, and $R_{\text {hole,out }}=3.3 a$ for $e=0.75$ (model 3$)$. The results are summarized in Table 3 and are plotted in Fig. 3 as the pink area in the sensitive curves. This area represents the extension within the disk hole/gap where stellar companions could exist according to these models and for a face-on geometry. The upper limit of this region is given by the circular orbit (model $1, d_{\mathrm{S}}=R_{\text {hole,out }} / 1.7$ ) and the lower limit by the periapsis of the most eccentric orbit (model $3 ; d_{\mathrm{S}}=a(1-e)=R_{\text {hole,out }} / 3.3(1-0.75)$ ). The corresponding orbital period $P$ was computed assuming Keplerian rotation ${ }^{6}$ and $m=0.5 M_{\star}$ at the location where companions are expected.

\subsubsection{Projection effects}

In this section we evaluate the orbit projection effects on our results on the basis of the disk inclinations $i$ reported in the literature for the five sources, assuming the binary is coplanar with the disk. Companions in nearly edge-on systems are likely to be occulted by the primary or by the disk itself. The gap properties also remain unchanged until the disk is nearly edge-on and starts to obscure the central star (Brown et al. 2007). Assuming a circular orbit for simplicity (we could not access the eccentricity of a detected companion with just one epoch images, anyway) and polar coordinates with the origin of the referential at the primary, the projected orbit can be computed with $x_{\text {proj }}=a \cos \theta$ and $y_{\text {proj }}=a \sin \theta \cos i$, where $a$ is the semi-major axis of the real orbit, $i$ the inclination and $\theta$ the angle between the position vector and the $x$-axis. The projected distance between the primary and secondary is determined by $d_{\text {proj }}=\left(x_{\text {proj }}^{2}+y_{\text {proj }}^{2}\right)^{1 / 2}$ at any point in the orbit. The results are listed in Table 3 and, as expected, the projection effects are more significant for the more inclined disks HR 4796A and T Cha.

In our case, a non-detection of the companion would occur for projected distances shorter than 0 .' $^{\prime} 1$, the detection limit in radii of the NACO images. Confining the analysis to the first quadrant $\left(0^{\circ}<\theta<90^{\circ}\right)$, we can determine the angle $\theta$ for which $d_{\text {proj }}<0$.' 1 and predict the percentage of the orbit

${ }^{6} P^{2}=\frac{4 \pi^{2}}{G\left(M_{\star}+m\right)} a^{3}$, where $G$ is the gravitational constant, $M_{\star}$ the mass of the primary and $m$ the mass of the secondary. 
Table 3. Predicted binary signatures from the models of Artymowicz \& Lubow (1994), projection effects caused by the inclination of the orbit, and hole/gap range where stellar/substellar companions can be ruled out from the NACO images.

\begin{tabular}{|c|c|c|c|c|c|c|c|c|c|c|c|c|}
\hline \multirow[t]{2}{*}{ Star } & \multirow{2}{*}{$\begin{array}{r}R_{\text {hole,out }} \\
(\mathrm{AU})\end{array}$} & \multicolumn{2}{|c|}{ Model $1^{1}$} & \multicolumn{2}{|c|}{ Model $2^{2}$} & \multicolumn{2}{|c|}{ Model $3^{3}$} & \multirow{2}{*}{$\begin{array}{c}i \\
(\operatorname{deg})\end{array}$} & \multicolumn{2}{|c|}{ Proj. effects ${ }^{4}$} & \multirow{2}{*}{$\begin{array}{c}\Delta R_{\text {stellar }}{ }^{5} \\
(\mathrm{AU})\end{array}$} & \multirow{2}{*}{$\begin{array}{c}\Delta R_{\text {substellar }}{ }^{6} \\
(\mathrm{AU})\end{array}$} \\
\hline & & $\begin{array}{c}d_{\mathrm{S}} \\
(\mathrm{AU})\end{array}$ & $\begin{array}{c}P \\
(\mathrm{yr})\end{array}$ & $\begin{array}{c}d_{\mathrm{S}} \\
(\mathrm{AU})\end{array}$ & $\begin{array}{c}P \\
(\mathrm{yr})\end{array}$ & $\begin{array}{c}d_{\mathrm{S}} \\
(\mathrm{AU})\end{array}$ & $\begin{array}{c}P \\
(\mathrm{yr})\end{array}$ & & $\begin{array}{l}d_{\text {proj }}{ }^{*} \\
(\mathrm{AU})\end{array}$ & $\begin{array}{l}d_{\text {proj }}{ }^{* *} \\
(\mathrm{AU})\end{array}$ & & \\
\hline DoAr 21 & 100 & 59 & 276 & $29-48$ & $95-202$ & $7.6-53$ & $13-235$ & $<45$ & $42-59$ & $71-100$ & $12.2-100$ & $31-100$ \\
\hline HD 135344B & 39 & 23 & 69 & $11-19$ & $23-52$ & $3-21$ & $3-60$ & 21 & $21-23$ & $36-39$ & $14-39$ & $37-39$ \\
\hline HR 4796A & 69 & 41 & 136 & $20-33$ & $46-98$ & $5-37$ & $6-116$ & 76 & $10-41$ & $17-69$ & $7.3-69$ & $13-69$ \\
\hline T Cha & 15 & 8.8 & 17.4 & $4.3-7.2$ & $6-13$ & $1.1-7.9$ & $0.8-15$ & 60 or 75 & $2.3-8.8$ & $4-15$ & $10.8-15$ & - \\
\hline TW Hya & 4.3 & 2.53 & 4.2 & $1.25-2.1$ & $1.5-3.2$ & $0.33-2.3$ & $0.2-3.7$ & 4.3 & $2.52-2.53$ & $4.29-4.3$ & - & - \\
\hline
\end{tabular}

Notes. ${ }^{(1)}$ Model 1 (circular orbit): $e=0, R_{\text {hole,out }}=1.7 a$ and the distance of the secondary relative to the primary is $d_{\mathrm{S}}=a=R_{\mathrm{hole}, \text { out }} / 1.7$. (2) Model 2: $e=0.25, R_{\text {hole,out }}=2.6 a$ and $a(1-e)<d_{\mathrm{S}}<a(1+e)$ or $0.29 R_{\text {hole,out }}<d_{\mathrm{S}}<0.48 R_{\text {hole,out. }}{ }^{\text {(3) }}$ Model 3: $e=0.75, R_{\text {hole,out }}=3.3 a$ and $a(1-e)<d_{\mathrm{S}}<a(1+e)$ or $0.076 R_{\text {hole,out }}<d_{\mathrm{S}}<0.53 R_{\text {hole,out }}{ }^{(4)}$ Binary coplanar with the disk $\left(i_{\mathrm{S}}=i_{\text {disk }}\right)$, circular orbit $(e=0), x_{\text {proj }}=a$ cos $\theta$, $y_{\text {proj }}=a \sin \theta \cos i$ and $d_{\text {proj }}=\left(x_{\text {proj }}^{2}+y_{\text {proj }}^{2}\right)^{1 / 2} ; d_{\text {proj }}(\max )=a$ for $\theta=0^{\circ}$ and $d_{\text {proj }}(\min )=a \cos i$ for $\theta=90^{\circ}$. Computed for ${ }^{*} a=R_{\text {hole,out }} / 1.7$ and ${ }^{* *} a=R_{\text {hole,out. }}{ }^{(5)}$ Range of disk hole radii, starting at 0 '. 1 from the primary, in which stellar binary companions can be ruled out on basis of the VLT/NACO images and using the BT-DUSTY evolutionary models. ${ }^{(6)}$ Range of disk hole radii in which substellar binary companions can be ruled out from this work.

we could effectively detect with our NACO observations. We performed this exercise for two values of the semi-major axis, $a=R_{\text {hole,out }} / 1.7$, and $a=R_{\text {hole,out }}$. The results show that a nondetection of the companion owing to projected distances shorter than 0 .' 1 could exist only for T Cha for $a=R_{\text {hole, out }}=15$ AU. At a distance of $108 \mathrm{pc}\left(0{ }^{\prime}{ }^{\prime} 1 \sim 10.8 \mathrm{AU}\right)$ and for disk inclinations $i=75^{\circ}$ or $60^{\circ}$ reported in the literature, a non-detection could occur if $\theta=45^{\circ}$ or $50^{\circ}$ (1st quadrant) or when $45-50^{\circ}<\theta<$ $225-230^{\circ}$ and $225-230^{\circ}<\theta<315-320^{\circ}$, for the entire orbit. Therefore, we would access 50 to $55 \%$ or 19.5 to $21.5 \mathrm{yr}$ of a total orbit of $39 \mathrm{yr}$ for a distance of $15 \mathrm{AU}$ from the primary and $m=0.5 M_{\star}$. If $a=R_{\text {hole }} / 1.7=8.8 \mathrm{AU}$, the NACO images are no longer sensitive to the distances involved. This is also the case for TW Hya, for which 0 .' $^{\prime} \sim 5.5 \mathrm{AU}$ and $R_{\text {hole,out }}=4.3 \mathrm{AU}$.

\section{Discussion: hole/gap formation mechanisms}

The origin of transitional disks is still under debate with several theories developed to explain inner opacity holes and gaps in protoplanetary disks, including i) magneto-rotational instability (MRI; Chiang \& Murray-Clay 2007), ii) photoevaporation by the central star (Clarke et al. 2001; Alexander et al. 2006; Gorti et al. 2009), iii) dust removal by coagulation (Tanaka et al. 2005; Dullemond \& Dominik 2005) and iv) dynamical clearing by unresolved companions, both stars (Artymowicz \& Lubow 1994), and giant planets (Quillen et al. 2004; Varnière et al. 2006).

The MRI mechanism operates in ionized gas and hence, at all disk surface layers ionized by direct X-rays from the central star, including the inner disk wall. Although this still allows accretion onto the star, MRI cannot explain the remnant optically thick disk observed in "pre-transitional" disks. Current photoevaporation models require a maximum total disk mass of $\sim 5 M_{\text {Jup }}$ and negligible accretion rate, unless the disk is unusually inviscid or large (>100 AU). Extreme-UV (EUV), far-UV (FUV) photons and X-rays from the central star heat and ionize the circumstellar hydrogen, generating a disk evaporative wind beyond some critical radius. At disk early-evolutionary stages, the accretion rate dominates over the evaporation rate. For photoevaporation to hold, the accretion rate has to fall below the wind rate and, once this happens, the photoevaporative wind can open a gap in the gas disk. The dust in the inner disk is then rapidly removed (on a viscous timescale of typically less than $10^{5} \mathrm{yr}$ ), forming the inner hole, and the entire disk dissipates very quickly through photoevaporation. Computed disk lifetimes are $\sim 4$ Myr for $M_{\star} \leq 3 M_{\odot}$ and less than 1 Myr for more massive stars (Gorti et al. 2009). The inner disk drains away entirely before the gap can grow beyond 1-10 AU, and therefore photoevaporation cannot explain the inner dust disks and large inner holes observed in many transitional objects. Grain growth can also produce opacity holes but is a strong function of the radius. It is more efficient at disk small radii where the surface density is higher and the dynamical timescales are shorter and do not affect accretion (of the gas). Unfortunately, is not possible to detect grains larger than a few millimeter and, therefore, this mechanism was not observationally confirmed in the last years. And finally, as discussed in Sect. 3.2, stellar/substellar companions or giant planets of 1 to a few $M_{\text {Jup }}$ can open gaps and inner holes through tidal interaction with the disk. While a stellar companion is expected to almost suppress accretion, an embedded planet of $\sim 1 M_{\text {Jup }}$ has only a modest effect on the disk accretion rate, reducing it by a factor of $\leq 10$ inside the planet's orbit compared to that from outside, and has little effect on the total disk mass (Lubow \& D'Angelo 2006). And, for a given disk mass, the more massive the planet and the less viscous the disk, the lower the accretion rate (Alexander \& Armitage 2007). Lubow et al. (1999) proposed that a planet of $10 M_{\text {Jup }}$ or more could halt stellar accretion. Nevertheless, a few observed close stellar/substellar binaries have been reported to be accreting: DQ Tau (Carr et al. 2001), CS Cha (Espaillat et al. 2007) and, more recently, T Cha (Huélamo et al. 2011; Olofsson et al. 2011). Factors other than the presence and mass of a close companion must determine accretion onto the star and hence, the accretion rate alone cannot be used to infer or discard the presence of potential companions. The viscosity and scale height of the disk and eccentricity of the orbit may contribute to maintain the accretion across the disk gap/hole even for stellar companions (Artymowicz \& Lubow 1996).

Alexander \& Armitage (2007) proposed a simple observational diagnostic to distinguish between photoevaporation and giant planet-induced inner holes that relies on the relative values of the the total disk mass $\left(M_{\text {disk }}\right)$ and stellar accretion rate $\left(\dot{M}_{\text {acc }}\right)$. More recently, Cieza et al. (2010) used optical-to-millimeter observations of a sample of 26 transitional disks in the Ophiuchus molecular cloud $(d \sim 125 \mathrm{pc})$ to derive the disk parameters 
and investigate the mechanisms that potentially create their inner holes. In addition to the disk mass and accretion rate, the authors also consider the fractional disk luminosity $\left(L_{\text {disk }} / L_{\star}\right)$ and parameters from the SED. According to their criteria, grain-growth-dominated disks have high-accretion rates $\left(\dot{M}_{\text {acc }} \geq\right.$ $10^{-8} M_{\odot} \mathrm{yr}^{-1}$ ) and large disk masses because this mechanism mostly operates in the disk inner region, whereas most of the mass resides at larger radii. Photoevaporating or photoevaporated disks should have a small disk mass $\left(M_{\text {disk }}<2.5 M_{\text {Jup }}\right)$ and negligible accretion $\left(<10^{-11} M_{\odot} \mathrm{yr}^{-1}\right)$, and if in addition the fractional disk luminosity $L_{\mathrm{disk}} / L_{\star}<10^{-3}$, the disk could be already in the debris evolutionary stage regardless the origin of the inner hole. Good candidates for ongoing giant planet formation are disks with large mass $\left(1.5-11 M_{\text {Jup }}\right)$ and low accretion rate $\left(\sim 10^{-10}-10^{-9} M_{\odot} \mathrm{yr}^{-1}\right)$, while disks with small mass $\left(<1.5 M_{\text {Jup }}\right)$ and higher accretion $\left(10^{-9.3}-10^{-7.3} M_{\odot} \mathrm{yr}^{-1}\right)$ could have formed a giant planet in a recent past because most of the disk mass has already been depleted. Close binaries are ruled out on the basis of the Artymowicz \& Lubow (1994) models $\left(R_{\text {hole, out }} \sim 2 a\right)$.

Following these criteria and using the disk parameters in Table 2, we tentatively classify the five transitional disks in our sample on the basis of the mechanisms that potentially create their inner opacity hole/gap. DoAr 21 was classified as a possible circumbinary or debris disk by Cieza et al. (2010) (source 12 in their catalog). HD 135344B, T Cha, and TW Hya are good candidates for ongoing giant planet-formation because of their large disk masses and modest accretion rates. HR 4796A could be a photoevaporated, debris, or a planetary disk owing to its very low mass, dust ring structure and no reported accretion rate. Nevertheless, this classification is just a best guess given the available data and the uncertainty associated to all parameters, both observational or derived from the models. Additionally, the hole/gap clearing mechanisms are not all mutually exclusive. Two or more mechanisms can operate at the same time (for example, grain growth and MRI, or planet formation and photoevaporation) or sequentially (once the gap has formed by one mechanism, others can rapidly remove the inner disk), contributing to the present observed transitional evolutionary state of the disk. Transitional disks seem to represent a highly heterogeneous group of objects exhibiting a wide range of masses, accretion rates, and SED morphologies although, overall, they tend to have smaller masses and lower accretion rates than disks without holes (Cieza et al. 2010). To conclude, disk dissipation does not seem to follow a standard evolutionary path, but instead many different paths are possible to which different mechanisms contribute.

The VLT/NACO images presented here provide an observational input to the last proposed mechanism by ruling out the presence of stellar companions within part (beyond 0.'1) of the inner hole/gap region of four disks in our sample and down to the planetary mass regime for DoAr 21 and to the substellar mass regime for HD 135344B and HR 4796A. The exception is TW Hya, for which the NACO images cannot resolve the inner hole of just $4.3 \mathrm{AU}$, or 78 mas for a distance of $55 \mathrm{pc}$. The results in Fig. 3 (pink bars in the plots) and Table 3 show that our observations are sensitive to part of the region where binaries are expected to exist from the models of Artymowicz \& Lubow (1994) for HD 135344B and almost this entire region in the case of HR 4796A and DoAr 21. With the proviso of possible occultations, we do not report any detection of companions beyond 0.1 from the primary for the five transitional disks. Therefore, the results in this paper further constrain the suite of potential explanations for their origin and toward the exciting possibility that giant planets are currently forming inside their inner holes/gaps. Nevertheless, the fraction of transitional disks that are in fact tight binaries still remains to be established. Common adaptive optics observations (on 8 to $10 \mathrm{~m}$ class telescopes) cannot constrain the inner 0.11 region from the primary, or radii of less than 5 to $15 \mathrm{AU}$ for most transitional disks. Additional surveys using different techniques such as sparse aperture masking (SAM) with adaptive optics instruments (the one leading to the detection of the potential substellar companion of T Cha reported in Huélamo et al. 2011) and radial velocity studies, should be performed to probe the inner most regions and firmly establish the fraction of transitional disks that are in fact close binaries. Finally, the Atacama Large Millimeter Array (ALMA) with its 1-3 AU spatial resolution and high sensitivity will be capable of resolving young planetary systems, detecting and determining the properties of close companions (both planets and stars) and of the dust and gas through out the disk. Planet and inner hole/gap formation scenarios will be confirmed or ruled out.

The following sections summarize the results regarding the presence of potential companions for each individual source from previous studies and this work.

\section{$\operatorname{DoAr} 21$}

Jensen et al. (2009) summarizes the properties of this object. Gemini sub-arcsecond resolution 9-18 $\mu \mathrm{m}$ images show little or no excess mid-IR emission within $100 \mathrm{AU}$ from the star. But beyond that the emission extends over several arcsec (or hundreds of AU) and is quite asymmetric, showing a half-ring structure of higher emission at $11^{\prime \prime} 1$ from the star to the NW. This feature is bright in PAHs emission at 8.6 and $11.3 \mu \mathrm{m}$, $\mathrm{H}_{2}$ emission at $2.12 \mu \mathrm{m}$ (the $1-0 \mathrm{~S}(1)$ ro-vibrational line) and continuum emission at $18 \mu \mathrm{m}$, and very different from what is expected from a disk. DoAr 21 is a particular object different from other pre-main sequence stars (PMS) because it is very young, a very strong emitter of (hard) X-rays and shows no accretion. Based on the estimated FUV and X-ray flux at the position of the ring ( $\sim 130 \mathrm{AU})$ and the proper motion of DoAr 21 $\left(9.5 \pm 1.3 \mathrm{~km} \mathrm{~s}^{-1}\right.$ westward), Jensen et al. (2009) proposed this emission to be associated with a small photodissociation region (PDR) resulting from the interaction of the radiation field with denser material from the Ophiuchus cloud in the vicinities of the star and not with a circumstellar (or circumbinary) disk. The half-arc shape would form because the material is swept up by the stellar motion. The $\mathrm{H}_{2}$ emission was spatially resolved with the AO-assisted IFU instrument SINFONI at the VLT as an arc of material circling the star at radii between $0 .^{\prime} 6$ (73 AU) and $1^{\prime \prime} .8$ (220 AU) and position angles of $-40^{\circ}$ to $170^{\circ}$ by Hogerheijde et al. (2011), who proposed several explanations for the asymmetric emission. The derived $\mathrm{H}_{2}$ line ratios were consistent with thermally excited gas by X-rays ( $T=1000$ $2000 \mathrm{~K}$ ) and not with FUV fluorescence. The most likely scenarios include illumination of unrelated cloud material or material resultant from the collision between two (proto)planets and the migration of a substellar/planetary companion to the ring region (73-220 AU) that could disrupt a previously stable disk. The last scenario was ruled out by their SINFONI images down to a mass limit of $5 M_{\mathrm{Jup}}$, by assuming an age of $0.3 \mathrm{Myr}$ for DoAr 21 . However, an unseen planet of a few $M_{\text {Jup }}$ could still explain the observations. Future epoch observations are necessary to 
determine if this material is orbiting the star (in a disk) or if is traveling through the cloud together with DoAr 21, confirming the PDR scenario.

VLBA observations by Loinard et al. (2008) show DoAr 21 as a binary with 5 mas $(0.6 \mathrm{AU})$ projected separation and estimated semi-major axis of 1-2 AU, but no information is given on the mass or optical luminosity ratio. Assuming it to be an equalmass binary, evolutionary tracks predict for each star a mass of $\sim 1.8 M_{\odot}\left(\sim 3.6 M_{\odot}\right.$ for the binary $)$ and an age of $\sim 8 \times 10^{5} \mathrm{yr}$. Nevertheless, such a close companion is beyond the resolution of NACO.

As expected, the $\mathrm{H}_{2}$ emission was not recovered in our VLT/NACO $2.12 \mu \mathrm{m}$ images (the width of the $\mathrm{H}_{2}$ line in this source is of $2 \times 10^{-4} \mu \mathrm{m}$ and was detected with an integration time of $7 \mathrm{~s}$ and a $S / N$ of $\sim 10$ ). Taking into account the width of $0.022 \mu \mathrm{m}$ of the NB_2.12 NACO filter, one would have needed integration times 220 times longer (i.e., $25 \mathrm{~min}$ ) to achieve a similar $S / N$ with our observations. Despite failing to detect offsource emission at $2.12 \mu \mathrm{m}$, the NACO images confirm the absence of substellar/planetary companions in the arc region down to a mass of $15 M_{\text {Jup }}$ at $73 \mathrm{AU}$ and $6 M_{\text {Jup }}$ at $192 \mathrm{AU}$. Our NACO images conservatively exclude substellar objects $\left(m<75 M_{\text {Jup }}\right)$ at radii larger than $\sim 31 \mathrm{AU}(0.25)$ and of stellar companions within the extension of the circumstellar environment probed

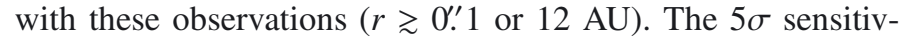
ity at $0 . ' 1$ is $3.1 \mathrm{mag}$ or $0.19 M_{\odot}$ for an assumed age of $1 \mathrm{Myr}$.

Cieza et al. (2010) collected nearly diffraction VLT/NACO/S13 images in the $J$ - and $K_{\mathrm{s}}$-bands ( $F W H M$ of 60-70 mas) from April to September 2009 of several transitional disks, including DoAr 21. A visual inspection of those images revealed no stellar companions beyond $\sim 8$ AU from the primary, which agrees with our results. Their detection limits at 0.1 separation, estimated from the $5 \sigma$ noise in the PSF subtracted images, have a median value of 3.1 mag corresponding to a flux ratio of 17. These authors classified DoAr 21 as a possible circumbinary or debris disk based on the disk parameters and no observed accretion (see discussion above). As proposed also by Jensen et al. (2009), the DoAr 21 disk must have almost entirely dissipated either through binary formation or photoevaporation. This scenario is indeed possible. An equal-mass eccentric binary with semi-major axis of 1-2 AU would tidally clear out an inner hole of 3 to $6 \mathrm{AU}$. Once the gap was opened, the high FUV and X-ray luminosity of the binary with mass of $\sim 3.6 M_{\odot}$ would photoevaporate the disk on a timescale of $\sim 10^{5}-10^{6} \mathrm{yr}$ (Gorti et al. 2009).

The NACO images of DoAr 21 are the only ones from the sample of five disks that are sensitive enough for a detection of objects less massive than $\sim 13 M_{\text {Jup }}$ that is, potential planets or low-mass brown dwarfs within the inner hole region of the disk and at radii larger than $\sim 76 \mathrm{AU}\left(0{ }^{\prime} 63\right)$.

\section{HD 135344B or SAO 206462}

Direct imaging at $880 \mu \mathrm{m}$ with the Submillimeter Array (SMA) by Brown et al. (2009) spatially resolved the disk and an inner hole extending to a radius of $39 \mathrm{AU}$, for a distance of $140 \mathrm{pc}$ to $\mathrm{HD} 135344 \mathrm{~B}$, in agreement with the value of $45 \mathrm{AU}$ derived from the SED best-fit model (Brown et al. 2007). This star has a wide companion at 20!'4 (Coulson \& Walther 1995). HD 135344B has been observed by the HST in coronographic mode with NICMOS $(1.1$ and $1.6 \mu \mathrm{m})$ and STIS and no companions were found (Grady et al. 2005, 2009). The $5 \sigma$ detection sensitivity limits show the non-existence of stellar companions within $\sim 0.1$ to $1 \operatorname{arcsec}(\sim 14$ to $140 \mathrm{AU})$ from the star.
Pontoppidan et al. (2008) detected CO emission at $4.7 \mu \mathrm{m}$ extending from 0.3 to $15 \mathrm{AU}$ from the primary with CRIRES at the VLT, arguing against stellar clearing, but in favor of a planetary mass companion orbiting at 10-20 AU. Spectroastrometry by Baines et al. (2006) also excludes stellar companions farther out than $0.42 \mathrm{AU}$. All these studies cannot exclude substellar objects within the disk (160 AU). The current NACO observations extend the previous results by excluding brown-dwarf-mass companions at orbits larger than $\sim 37 \mathrm{AU}\left(0 .{ }^{\prime} 27\right)$ and down to a mass limit of $\sim 19 M_{\text {Jup }}\left(8 \mathrm{mag}\right.$ in contrast) at $221 \mathrm{AU}$. At $0{ }^{\prime} 1$ they are sensitive to stars more massive than $0.22 M_{\odot}$.

\section{HR 4796A}

Schneider et al. (2009) obtained high spatial resolution ( 70 mas) optical coronographic images of the HR 4796A debris dust ring with the Space Telescope Imaging Spectrograph (STIS) resolving emission from $\sim 69-87$ AU (0.'95-1.'.2) in radii (see Fig. 7 in their paper) and a displacement of 1.4 AU between the disk center and the star, measured along the ring major axis. These values are in accordance with previous observations at 1.1 and $1.6 \mu \mathrm{m}$ with the HST/NICMOS instrument (Schneider et al. 1999). The hole outer radius was first inferred from the SED by Jura et al. (1995) to be from 40 to 200 AU. HR 4796A is a visual binary having a M2.5-dwarf companion at 7.'7 or $560 \mathrm{AU}$ (Jura et al. 1993). More recently, a tertiary wide companion ( $\sim 3^{\prime}$ separation) was discovered from its X-ray emission and is a M4.5 spectral-type star (Kastner et al. 2008). No other companions have been detected in $H$ - and $K_{\mathrm{s}}$-band coronographic NACO imaging at projected separations in the range $0 .^{\prime} 1$ to $10^{\prime \prime}$ (Chauvin et al. 2010) and there are no radial velocity measurements for this source. Wyatt et al. (1999) concluded that a planet more massive than $10 M_{\text {Earth }}$ close to the inner edge of the ring would be required to explain the asymmetry seen in the dust emission. Such a low-mass object could not be detected with the observations presented here. Nevertheless, the NACO images discard the existence of stellar companions within the disk hole at radii larger than $\sim 7 \mathrm{AU}\left(0{ }^{\prime} 1\right)$ and more massive than $0.24 M_{\odot}$, and of substellar objects outwards of $\sim 13 \mathrm{AU}\left(0\right.$ ' $\left.^{\prime} 18\right)$. The mass detection limit at the hole outer radius (69 AU) and inside the dust ring (up to $87 \mathrm{AU}$ ) is $17 M_{\text {Jup }}$.

\section{TCha}

Radial velocity (RV) observations were performed for this source and the results are presented in Schisano et al. (2009). The authors report a radial velocity variation of $10 \mathrm{~km} \mathrm{~s}^{-1}$, albeit non-periodic. No companions for T Cha were detected at projected separations of 0 . $^{\prime} 1$ to $10^{\prime \prime}$ in diffraction-limited $H$ - and $K_{\mathrm{s}}$-band NACO images with Lyot-coronography (Chauvin et al. 2010). Recently, Huélamo et al. (2011) reported the presence of a potential substellar companion at a separation of $62 \pm 7$ mas $(6.7 \pm 1 \mathrm{AU})$ from $\mathrm{T}$ Cha detected in the $L^{\prime}$-band (contrast $\Delta L^{\prime}=$ $5.1 \pm 0.2 \mathrm{mag}$ ) with NACO and using sparse aperture masking (SAM). The object was not detected in the authors' $K_{\mathrm{s}}$-band data up to a $3 \sigma$ contrast limit of $5.2 \mathrm{mag}$. The corresponding $5 \sigma$ contrast limit is $\sim 4.65 \mathrm{mag}$ or $0.12 M_{\odot}$ for the BT-DUSTY model adopted here and is indicated in Fig. 3 as the blue vertical bar in the T Cha sensitivity plot. A lower limit of $50 M_{\text {Jup }}$ was chosen following Fig. 4 in Huélamo et al. (2011) paper. This detection is 
well within the T Cha disk hole/gap and inside the binary range predicted by the models of Artymowicz \& Lubow (1994) ${ }^{7}$.

Although we could not have detected such an inner low-mass object, these observations support ours and the Chauvin et al. (2010) results by ruling out stellar companions outward of 0 ' $^{\prime} 1$ from the primary. The NACO images of T Cha exclude stellar companions brighter than $2.2 \mathrm{mag}$ in contrast $\left(0.29 M_{\odot}\right)$ at a projected separation of 0 .' 1 to $2.9 \mathrm{mag}\left(0.25 M_{\odot}\right)$ at $15 \mathrm{AU}$, or within the disk inner hole. They are not sensitive enough for the detection of substellar binaries within the whole extension of the disk. The maximum attainable contrast is $4.7 \mathrm{mag}$, corresponding to a $5 \sigma$ sensitivity of $0.12 M_{\odot}$.

\section{TW Hya}

Detailed modeling of the SED of the star predicted a disk hole/gap outer radius of $\sim 4 \mathrm{AU}$ (Calvet et al. 2002) later on confirmed with Very Large Array (VLA) observations at $7 \mathrm{~mm}$ by Hughes et al. (2007). The data resolved the hole in the dust disk and revealed a bright inner rim associated with the disk edge being directly illuminated by the star. Setiawan et al. (2008) reported a $10 M_{\text {Jup }}$ companion at $0.04 \mathrm{AU}$ from the star through RV observations in the optical regime. Huélamo et al. (2008) carried out near-IR high-resolution observations of the source with the CRyogenic high-resolution InfraRed Echelle Spectrograph (CRIRES) mounted on the VLT and found no trace of this companion. Spectro-astrometric imaging of the $4.7 \mu \mathrm{m}$ rovibrational lines of CO by Pontoppidan et al. (2008) detected gas in the TW Hya disk hole between 0.1 and 1.5 AU. Based on this result, the authors suggest that the presence of a $\sim 16 M_{\text {Jup }}$ body could be clearing the dust and rule out photoevaporation as the hole formation mechanism. They further explain that if the 4 AU hole is created by a planet, it should be placed in an orbit of 1-2 AU. The present NACO observations cannot resolve these inner disk regions.

Direct coronographic imaging of the disk in scattered light at 1.1 and $1.6 \mu \mathrm{m}$ with the HST/NICMOS instrument was collected by Weinberger et al. (2002). The scattering profile indicates a flared disk extending from $\sim 20$ to 230 AU. No point sources were detected with a $3 \sigma$ significance from $\sim 0$.' 54 to $4^{\prime \prime}$ (or 30 to $220 \mathrm{AU}$ ) down to a mass of $\sim 10 M_{\text {Jup }}$ at $\sim 0.63$ (35 AU) and $3 M_{\text {Jup }}$ at 1'.52 (85 AU). Additionally, TW Hya was included in an adaptive optics imaging survey with the Keck II $10 \mathrm{~m}$ telescope by Brandeker et al. (2003). The star was observed in the $H$-band and reached a $5 \sigma$ contrast sensitivity of $\sim 2$ mag at a separation of 0.1 . No companions were detected between 0.042 (the diffraction limit in the $H$-band) and $1 . \prime 6$, or from 2.3 to $88 \mathrm{AU}$.

\footnotetext{
7 Assuming the companion is coplanar with the disk, which has an inclination of $60^{\circ}$ to $75^{\circ}$ reported in the literature, and a circular orbit, we can put some constraints on the orbit of the potential companion by considering the two extreme cases: 1) $\theta=0^{\circ}$ and the distance primarysecondary equals the binary semi-major axis, $d_{\mathrm{s}}=a=6.7 \mathrm{AU}$, and 2) $\theta=90^{\circ}$ and $d_{\mathrm{s}}=a \cos i=6.7 \mathrm{AU}$. In 1) the potential companion is at its largest projected separation at the moment and will reach the shortest separation of 1.7 to $3.3 \mathrm{AU}$ when $\theta=90^{\circ}$ for an inclination varying between $60^{\circ}$ and $75^{\circ}$. The orbital period varies between $13.6 \mathrm{yr}$ (for $m=0.12 M_{\odot}$ ) and $14 \mathrm{yr}$ (for $m=50 M_{\text {Jup }}$ ). In 2) the companion is at its shortest projected separation in the present and the semi-major axis of the orbit $a$ can be derived assuming an inclination of the orbit $i$ : for $i=60^{\circ}, a=13.4 \mathrm{AU}$ and for $i=75^{\circ}, a=26 \mathrm{AU}$. The orbital period varies between $\sim 39 \mathrm{yr}\left(i=60^{\circ}\right)$ and $\sim 105 \mathrm{yr}\left(i=75^{\circ}\right)$. Future epoch observations will better constrain the orbit of the potential companion by providing the inclination and the eccentricity.
}

The results derived here from the NACO imaging further complement and support the previous studies by excluding stellar binaries more massive than $0.11 M_{\odot}$ ( 3 mag in contrast) outward of $\sim 5.5 \mathrm{AU}$ from the primary (the inner detection limit of the observations or 0. '1), brown-dwarf-mass companions outward of $7 \mathrm{AU}\left(0 .{ }^{\prime} 13\right)$ and planetary mass companions outward of $\sim 13 \mathrm{AU}(0.24)$. We reach the maximum sensitivity of $7 M_{\text {Jup }}$, or 8 mag in contrast from 87 AU onward.

A recent paper by Vacca \& Sandell (2011) based on nearinfrared spectroscopic data revises the spectral type and age of TW Hya to M2.5 (instead of K7) and 3 Myr (instead of 8-10 Myr). Under the assumption of a younger age, the NACO images are now sensitive to substellar mass companions within the whole disk region probed $\left(0 . ' 1\right.$ to $\left.7{ }^{\prime \prime} 0\right)$ and to planetary-mass objects beyond $\sim 10 \mathrm{AU}(0 . ' 18)$.

\section{Conclusions}

This paper addresses one possible origin for inner holes or cleared gaps in protoplanetary disks. These holes/gaps are seen as potential signposts of planet formation but other explanations are possible. Here we investigate the mechanism of tidal truncation by close binaries as the possible or alternate explanation for the origin of the inner holes/gaps observed in five transitional disks. The conclusions are that:

1. With the proviso of possible unfavorable projections, the VLT/NACO images rule out the presence of binary companions from $0 . ' 1$ to $\sim 7^{\prime \prime}$ in all five transitional disks and down to a $5 \sigma$ detection limit of 2.2-4.9 mag in contrast for the inner region (at $0{ }^{\prime} 1$ ) and 7.7-9.3 mag (4.7 mag in the case of $\mathrm{T}$ Cha) for the outer region (beyond $11^{\prime \prime} 0$ ).

2. For the transitional disks DoAr 21, HD 135344B, HR 4796A, and T Cha, the VLT/NACO observations resolve part of the inner hole/gap region of the disk and can potentially discard binaries down to a certain mass limit as the main explanation for the inner opacity cavities. For these objects, our results reduce the suite of potential mechanisms that create the holes to closer or lower-mass companions, giant planet formation, efficient grain growth or photoevaporation (for DoAr 21 and HR 4796A).

Acknowledgements. The authors thank the anonymous referee for important comments, which improved this paper, and to Cátia Cardoso for fruitful discussion on binary dynamics. Support in the preparation of this publication was provided by the ESA Science Faculty. This publication makes use of data products from the Two Micron All Sky Survey, which is a joint project of the University of Massachusetts and the Infrared Processing and Analysis Center/California Institute of Technology, funded by the National Aeronautics and Space Administration and the National Science Foundation. This work has made use of the Vizier Service provided by the Centre de Données Astronomiques de Strasbourg, France (Ochsenbein et al. 2000). Image Reduction and Analysis Facility (IRAF) is distributed by the National Optical Astronomy Observatory (NOAO), which is operated by the Association of Universities for Research in Astronomy Inc. (AURA), under cooperative agreement with the National Science Foundation (NSF).

\section{References}

Akeson, R. L., Millan-Gabet, R., Ciardi, D. R., et al. 2011, ApJ, 728, 96 Alcala, J. M., Covino, E., Franchini, M., et al. 1993, A\&A, 272, 225 Alexander, R. D., \& Armitage, P. J. 2007, MNRAS, 375, 500

Alexander, R. D., Clarke, C. J., \& Pringle, J. E. 2006, MNRAS, 369, 216 Allard, F., Hauschildt, P. H., Alexander, D. R., Tamanai, A., \& Schweitzer, A. 2001, ApJ, 556, 357

Allard, F., Homeier, D., \& Freytag, B. 2010 [arXiv: 1011. 5405] 
Andrews, S. M., Wilner, D. J., Hughes, A. M., Qi, C., \& Dullemond, C. P. 2009, ApJ, 700, 1502

Artigau, É., Biller, B. A., Wahhaj, Z., et al. 2008, in SPIE Conf., 7014

Artymowicz, P., \& Lubow, S. H. 1994, ApJ, 421, 651

Artymowicz, P., \& Lubow, S. H. 1996, ApJ, 467, L77

Baines, D., Oudmaijer, R. D., Porter, J. M., \& Pozzo, M. 2006, MNRAS, 367, 737

Barrado Y Navascués, D. 2006, A\&A, 459, 511

Bary, J. S., Weintraub, D. A., \& Kastner, J. H. 2002, ApJ, 576, L73

Besla, G., \& Wu, Y. 2007, ApJ, 655, 528

Beust, H., \& Dutrey, A. 2006, A\&A, 446, 137

Brandeker, A., Jayawardhana, R., \& Najita, J. 2003, AJ, 126, 2009

Brown, J. M., Blake, G. A., Dullemond, C. P., et al. 2007, ApJ, 664, L107

Brown, J. M., Blake, G. A., Qi, C., Dullemond, C. P., \& Wilner, D. J. 2008, ApJ, 675, L109

Brown, J. M., Blake, G. A., Qi, C., et al. 2009, ApJ, 704, 496

Burrows, A., Marley, M., Hubbard, W. B., et al. 1997, ApJ, 491, 856

Burrows, A., Hubbard, W. B., Lunine, J. I., \& Liebert, J. 2001, Rev. Mod. Phys., 73,719

Calvet, N., D'Alessio, P., Hartmann, L., et al. 2002, ApJ, 568, 1008

Calvet, N., D'Alessio, P., Watson, D. M., et al. 2005, ApJ, 630, L185

Carr, J. S., Mathieu, R. D., \& Najita, J. R. 2001, ApJ, 551, 454

Chabrier, G., Baraffe, I., Allard, F., \& Hauschildt, P. 2000, ApJ, 542, 464

Chauvin, G., Lagrange, A., Bonavita, M., et al. 2010, A\&A, 509, A52

Chiang, E., \& Murray-Clay, R. 2007, Nature Phys., 3, 604

Cieza, L., Padgett, D. L., Stapelfeldt, K. R., et al. 2007, ApJ, 667, 308

Cieza, L. A., Swift, J. J., Mathews, G. S., \& Williams, J. P. 2008, ApJ, 686, L115

Cieza, L. A., Schreiber, M. R., Romero, G. A., et al. 2010, ApJ, 712, 925

Clarke, C. J., Gendrin, A., \& Sotomayor, M. 2001, MNRAS, 328, 485

Coulson, I. M., \& Walther, D. M. 1995, MNRAS, 274, 977

D’Alessio, P., Hartmann, L., Calvet, N., et al. 2005, ApJ, 621, 461

Devillard, N. 1997, The Messenger, 87, 19

Dullemond, C. P., \& Dominik, C. 2005, A\&A, 434, 971

Dutrey, A., Guilloteau, S., Piétu, V., et al. 2008, A\&A, 490, L15

Eisner, J. A., Chiang, E. I., \& Hillenbrand, L. A. 2006, ApJ, 637, L133

Eisner, J. A., Doppmann, G. W., Najita, J. R., et al. 2010, ApJ, 722, L28

Espaillat, C., Calvet, N., D’Alessio, P., et al. 2007, ApJ, 670, L135

Fedele, D., van den Ancker, M. E., Acke, B., et al. 2008, A\&A, 491, 809

Furlan, E., Watson, D. M., McClure, M. K., et al. 2009, ApJ, 703, 1964

Gorti, U., Dullemond, C. P., \& Hollenbach, D. 2009, ApJ, 705, 1237

Grady, C. A., Woodgate, B. E., Bowers, C. W., et al. 2005, ApJ, 630, 958

Grady, C. A., Schneider, G., Sitko, M. L., et al. 2009, ApJ, 699, 1822

Guenther, E. W., Esposito, M., Mundt, R., et al. 2007, A\&A, 467, 1147

Herbig, G. H. 1978, Can Post-T Tauri Stars Be Found?, ed. L. V. Mirzoyan, 171

Herczeg, G. J., Cruz, K. L., \& Hillenbrand, L. A. 2009, ApJ, 696, 1589

Høg, E., Fabricius, C., Makarov, V. V., et al. 2000, A\&A, 355, L27

Hogerheijde et al., 2011, submitted

Huélamo, N., Figueira, P., Bonfils, X., et al. 2008, A\&A, 489, L9

Huélamo, N., Lacour, S., Tuthill, P., et al. 2011, A\&A, 528, L7

Hughes, A. M., Wilner, D. J., Calvet, N., et al. 2007, ApJ, 664, 536

Hughes, A. M., Andrews, S. M., Espaillat, C., et al. 2009, ApJ, 698, 131

Ireland, M. J., \& Kraus, A. L. 2008, ApJ, 678, L59

Jensen, E. L. N., Cohen, D. H., \& Gagné, M. 2009, ApJ, 703, 252

Jura, M., Zuckerman, B., Becklin, E. E., \& Smith, R. C. 1993, ApJ, 418, L37

Jura, M., Ghez, A. M., White, R. J., et al. 1995, ApJ, 445, 451

Kastner, J. H., Zuckerman, B., \& Bessell, M. 2008, A\&A, 491, 829
Lafrenière, D., Marois, C., Doyon, R., Nadeau, D., \& Artigau, É. 2007, ApJ, 660,770

Lenzen, R., Hartung, M., Brandner, W., et al. 2003, in SPIE Conf. Ser. 4841, ed. M. Iye, \& A. F. M. Moorwood, 944

Loinard, L., Torres, R. M., Mioduszewski, A. J., \& Rodríguez, L. F. 2008, ApJ, 675, L29

Lommen, D., Wright, C. M., Maddison, S. T., et al. 2007, A\&A, 462, 211

Lubow, S. H., \& D'Angelo, G. 2006, ApJ, 641, 526

Lubow, S. H., Seibert, M., \& Artymowicz, P. 1999, ApJ, 526, 1001

Marois, C., Lafrenière, D., Doyon, R., Macintosh, B., \& Nadeau, D. 2006, ApJ, 641,556

Masset, F. S., \& Papaloizou, J. C. B. 2003, ApJ, 588, 494

Merín, B., Brown, J. M., Oliveira, I., et al. 2010, ApJ, 718, 1200

Meyer, M. R., Backman, D. E., Weinberger, A. J., \& Wyatt, M. C. 2007, in Protostars and Planets V, ed. B. Reipurth, D. Jewitt, \& K. Keil, 573

Muzerolle, J., Allen, L. E., Megeath, S. T., Hernández, J., \& Gutermuth, R. A. 2010, ApJ, 708, 1107

Najita, J. R., Strom, S. E., \& Muzerolle, J. 2007, MNRAS, 378, 369

Oliveira, I., Pontoppidan, K. M., Merín, B., et al. 2010, ApJ, 714, 778

Olofsson, J., Benisty, M., Augereau, J., et al. 2011, A\&A, 528, L6

Perez-Becker, D., \& Chiang, E. 2011, ApJ, 727, 2

Piétu, V., Dutrey, A., Guilloteau, S., Chapillon, E., \& Pety, J. 2006, A\&A, 460, L43

Pontoppidan, K. M., Blake, G. A., van Dishoeck, E. F., et al. 2008, ApJ, 684, 1323

Pott, J., Perrin, M. D., Furlan, E., et al. 2010, ApJ, 710, 265

Qi, C., Ho, P. T. P., Wilner, D. J., et al. 2004, ApJ, 616, L11

Quillen, A. C., Blackman, E. G., Frank, A., \& Varnière, P. 2004, ApJ, 612, L137

Rieke, G. H., \& Lebofsky, M. J. 1985, ApJ, 288, 618

Rousset, G., Lacombe, F., Puget, P., et al. 2003, in SPIE Conf. 4839, ed. P. L. Wizinowich, \& D. Bonaccini, 140

Schisano, E., Covino, E., Alcalá, J. M., et al. 2009, A\&A, 501, 1013

Schneider, G., Smith, B. A., Becklin, E. E., et al. 1999, ApJ, 513, L127

Schneider, G., Weinberger, A. J., Becklin, E. E., Debes, J. H., \& Smith, B. A. 2009, AJ, 137, 53

Setiawan, J., Henning, T., Launhardt, R., et al. 2008, Nature, 451, 38

Sicilia-Aguilar, A., Hartmann, L., Calvet, N., et al. 2006, ApJ, 638, 897

Sicilia-Aguilar, A., Henning, T., Juhász, A., et al. 2008, ApJ, 687, 1145

Skrutskie, M. F., Dutkevitch, D., Strom, S. E., et al. 1990, AJ, 99, 1187

Spiegel, D. S., Burrows, A., \& Milsom, J. A. 2011, ApJ, 727, 57

Strom, K. M., Strom, S. E., Edwards, S., Cabrit, S., \& Skrutskie, M. F. 1989, AJ, 97, 1451

Tanaka, H., Himeno, Y., \& Ida, S. 2005, ApJ, 625, 414

Tanner, A., Beichman, C., Akeson, R., et al. 2007, PASP, 119, 747

Thalmann, C., Grady, C. A., Goto, M., et al. 2010, ApJ, 718, L87

Thi, W., Mathews, G., Ménard, F., et al. 2010, A\&A, 518, L125

Torres, C. A. O., Quast, G. R., da Silva, L., et al. 2006, A\&A, 460, 695

Torres, C. A. O., Quast, G. R., Melo, C. H. F., \& Sterzik, M. F. 2008, Young Nearby Loose Associations, ed. B. Reipurth, 757

Vacca, W. D., \& Sandell, G. 2011, ApJ, 732, 8

van Leeuwen, F. 2007, A\&A, 474, 653

Varnière, P., Blackman, E. G., Frank, A., \& Quillen, A. C. 2006, ApJ, 640, 1110

Webb, R. A., Zuckerman, B., Platais, I., et al. 1999, ApJ, 512, L63

Weinberger, A. J., Becklin, E. E., Schneider, G., et al. 2002, ApJ, 566, 409

Wyatt, M. C., Dermott, S. F., Telesco, C. M., et al. 1999, ApJ, 527, 918 